\title{
Synthesis Of PEG-Coated, Ultrasmall,
} Manganese-Doped Iron Oxide Nanoparticles With High Relaxivity For $T_{1} / T_{2}$ Dual-Contrast Magnetic Resonance Imaging

This article was published in the following Dove Press journal:

International Journal of Nanomedicine

\author{
Shilin Xiao \\ Xian Yu \\ Liang Zhang \\ Ya Zhang \\ Weijie Fan \\ Tao Sun \\ Chunyu Zhou \\ Yun Liu \\ Yiding Liu \\ Mingfu Gong (D) \\ Dong Zhang
}

Department of Radiology, Xinqiao Hospital, Army Medical University, Chongqing, People's Republic of China

Correspondence: Mingfu Gong; Dong Zhang

Department of Radiology, Xinqiao Hospital, Army Medical University, Xinqiao Street, Shapingba District,

Chongqing, People's Republic of China

Tel +862368763843

Fax +86236875 5306

Email hummer198625@163.com;

hszhangd@I63.com
Background: Beyond magnetic resonance imaging (MRI), which has been widely used clinically, molecular MRI (mMRI) can further provide qualitative and quantitative information at the cellular and molecular levels. However, the diagnostic accuracy may not be satisfactory via single-contrast mMRI due to some interferences in vivo. $T_{1} / T_{2}$ dual-contrast MRI using the same contrast agent (CA) could significantly improve the detection accuracy. Therefore, in this study, we fabricated poly(ethylene glycol) (PEG)-coated, manganesedoped iron oxide nanocomposites (Mn-IONPs@PEG) as $\mathrm{T}_{1} / \mathrm{T}_{2}$ dual-contrast CA, and evaluated its feasibility of $\mathrm{T}_{1} / \mathrm{T}_{2}$ dual-contrast MRI in vitro and in vivo.

Methods: Mn-IONPs were prepared by the thermal decomposition of iron-eruciate and manganese-oleate complexes and were coated with 1,2-Distearoyl-sn-glycero-3-phosphoethanolamine-N-(methoxy[polyethylene glycol]-2000) (DSPE-PEG 2000). The physicochemical properties and cytotoxicity of the Mn-IONPs were fully characterized, followed by MRI in vitro and in vivo.

Results: Ultrasmall $3 \mathrm{~nm}$-sized nanoparticles were successfully prepared and were identified using transmission electron microscopy (TEM), high-resolution TEM, and X-ray diffraction. After coating with DSPE-PEG, the Mn-IONPs@PEG displayed excellent hydrophilicity and good biocompatibility. Due to the manganese-doping and PEG coating, the Mn-IONPs@PEG showed good relaxivity in vitro. Especially, the Mn-IONPs@PEG coated with DSPE-PEG following a mass ratio to Mn-IONPs of 1:20 showed harmonious longitudinal relaxivity $\left(r_{1}=7.1 \mathrm{mM}^{-1} \mathrm{~s}^{-1}\right)$ and transversal relaxivity $\left(r_{2}=120.9 \mathrm{mM}^{-1} \mathrm{~s}^{-1}\right)$, making it a better candidate for $\mathrm{T}_{1} / \mathrm{T}_{2}$ dual-contrast mMRI. After administrated via a caudal vein, the Mn-IONPs@PEG can induce significant enhancement in both $T_{1}$-weighted and $T_{2}$-weighted $M R$ images and the time at 10 mins after injection was regarded as a suitable time for imaging because both the $T_{1}$ and $T_{2}$ enhancement were optimum at that time.

Conclusion: The obtained Mn-IONPs@PEG exhibited good $r_{1}$ and $r_{2}$ and was a reasonable candidate for $\mathrm{T}_{1} / \mathrm{T}_{2}$ dual-contrast mMRI.

Keywords: magnetic resonance imaging, manganese-doped iron oxide nanoparticles, $T_{1} / T_{2}$ dual-contrast, DSPE-PEG coating, high relaxivity

\section{Introduction}

In recent decades, magnetic resonance imaging (MRI) has played an irreplaceable role in medical diagnosis because of its efficient soft tissue contrast, high spatial resolution, lack of ionizing radiation, and unrestricted signal penetration depth. ${ }^{1,2}$ 
Particularly, molecular magnetic resonance imaging (mMRI) has extended beyond traditional MRI technology. mMRI can obtain qualitative and quantitative information about biological processes in vivo at the cellular and molecular levels, greatly expanding the research and application fields of MRI. ${ }^{3-5}$ In recent years, mMRI has been intensively studied in gene imaging, ${ }^{6}$ cell tracing, ${ }^{7}$ drug screening, ${ }^{8}$ and evaluating early diagnoses and therapeutic effects of disease. ${ }^{9,10}$ Contrast agent (CA) serving as the signal source of mMRI is the most important ingredient and has reasonably become a hot spot in the field of mMRI. ${ }^{11}$

Although many types of CA based on magnetic nanoparticles (NPs) with high longitudinal relaxivity $\left(r_{1}\right)^{12}$ or transverse relaxivity $\left(r_{2}\right)^{13}$ have been developed, singlecontrast mMRI still possesses insufficient sensitivity and specificity due to its innate properties. Transverse relaxation time $\left(\mathrm{T}_{2}\right)$ single-contrast CA usually results in MR signal loss and susceptibility artifacts. ${ }^{14}$ Additionally, the darker areas in MR $T_{2}$-weighted imaging $\left(\mathrm{T}_{2} \mathrm{WI}\right)$ induced by the $T_{2}$ single-contrast CA may be confused with other physiological or pathological low-signal regions, such as bleeding, calcification, and metal deposition, which will significantly reduce the sensitivity and specificity of mMRI. ${ }^{15}$ Similarly, some normal tissues (such as fatty tissue) with hyperintensity in MR longitudinal relaxation time $\left(\mathrm{T}_{1}\right)$-weighted imaging $\left(\mathrm{T}_{1} \mathrm{WI}\right)$ may be misunderstood as bright lesions enhanced by $T_{1} C A$. $T_{1} / T_{2}$ dualcontrast MRI using the same CA provides complementary information about a lesion to self-confirm and provide fault-free MR images, proving that it can significantly improve the detection accuracy. ${ }^{16,17}$

Thus far, multifarious CAs have been reported for $T_{1} / T_{2}$ dual-contrast MRI. A common strategy for the synthesis of $T_{1} / T_{2}$ dual-contrast $C A$ is to integrate $T_{1}$ and $T_{2}$ signal moieties as hybrid nanoparticles. ${ }^{17-23}$ However, due to the magnetic coupling effect, the enhancement generated by the $\mathrm{T}_{1}$ and $\mathrm{T}_{2}$ moieties will interact with each other, leading to the compromise of both $r_{1}$ and $r_{2}$. Embedding the $\mathrm{T}_{1}$ species into the $T_{2}$ species can parallel the electronic spins of paramagnetic $\mathrm{T}_{1} \mathrm{CA}$ with the direction of the magnetic field induced by the $T_{2} C A$, and reasonably enhance both the $T_{1}$ and $T_{2}$ relaxivity of the $T_{1} / T_{2}$ dual-contrast $C A .{ }^{16}$

Because of good biocompatibility, high relaxivity, and easy and versatile functionalization, superparamagnetic iron oxide nanoparticles (IONPs) have been extensively studied and have emerged as promising $\mathrm{T}_{2} \mathrm{CA} .{ }^{24}$ Benefiting from the size-dependent contrast, the ultrasmall IONPs also have demonstrated satisfactory $r_{1}$, making them alternative $T_{1} / T_{2}$ dual-contrast CA. ${ }^{25,26}$ Nevertheless, the increased $T_{1}$ relaxivity of the ultrasmall IONPs is the result of the consumption of the size, resulting in a relatively small $r_{2}$. Thus, ultrasmall IONPs may be not optimal as $T_{1} / T_{2}$ dual-contrast CA.

Divalent manganese ions $\left(\mathrm{Mn}^{2+}\right)$ possess five unpaired electrons and are of higher paramagnetism, and $\mathrm{Mn}^{2+}$ doping has been proven to be an effective route to improve the $r_{1}$ of ultrasmall IONPs. ${ }^{12,27,28}$ Additionally, doped $\mathrm{Mn}^{2+}$ with a higher magnetic moment $\left(\mu_{B}=5.92\right)$ can occupy both the tetrahedral $\left(\mathrm{T}_{\mathrm{d}}\right)$ and octahedral holes $\left(\mathrm{O}_{\mathrm{h}}\right)$ in the crystal lattices, resulting in a special mixed spinel structure, a larger saturation magnetization (Ms), and a high $r_{2}$ of manganesedoped IONPs (Mn-IONPs). ${ }^{2,29}$ Moreover, based on the embedding rationale, the doped $\mathrm{Mn}^{2+}$ and ultrasmall IONPs show synergetic enhancement, which will further improve both $r_{1}$ and $r_{2}$ of Mn-IONPs. Therefore, Mn-IONPs could be better candidates as dual-contrast $\mathrm{CA}$.

Usually, the hydrophobic inorganic nanoparticles need to be modified with either natural macromolecules or synthetic polymers for hydrophilia and further functionalization for biomedical applications. 1,2-Distearoyl-sn-glycero-3-phosphoethanolamine-N-(methoxy [polyethylene glycol]) (DSPE-PEG), with various advantages such as a low critical micellar concentration (CMC), excellent biocompatibility, and long blood circulation time has been diffusely employed for surface modification. More importantly, it was reported that DSPE-PEG can significantly improve $r_{2}$ of the IONPs via the immobilization of nearby water molecules by forming hydrogen bonds. ${ }^{30,31}$

Herein, Mn-IONPs were prepared through the dynamic simultaneous thermal decomposition (DSTD) method. Strikingly, after coating with DSPE-PEG, the obtained hydrophilic manganese-doped iron oxide nanocomposites (Mn-IONPs@PEG) showed both good $r_{1}$ and $r_{2}$ in vitro and in vivo, demonstrating that these nanocomposites comprising ultrasmall Mn-IONPs and DSPE-PEG could be ideal candidates for use as $T_{1} / T_{2}$ dual-contrast CA.

\section{Materials And Methods}

\section{Materials}

Oleyl alcohol and dibenzyl ether were purchased from Aldrich Chemical Co. (Saint Louis, MO, USA). Absolute ethyl alcohol was obtained from Chongqing Chuandong Chemical Co. Ltd. (Chongqing, China). Hexane and tetrahydrofuran (THF) were purchased from Adamas Reagent Co. Ltd. (Shanghai, China). DSPE-PEG2000 was obtained 
from Shanghai Ponsure Biotechnology Co. Ltd. (Shanghai, China). Dulbecco's Modified Eagle's Medium (DMEM) was purchased from Life Technologies Co. (Saint Louis, MO, USA). Fetal bovine serum (FBS) was obtained from Zhejiang Tianhang Biotechnology Co. Ltd. (Zhejiang, China). Penicillin/streptomycin solution and L-glutamine were purchased from Beyotime Biotechnology Co. Ltd. (Shanghai, China). Cell Count Kit-8 (CCK-8) was purchased from Boster Biological Technology Co. Ltd. (Wuhan, China). All of these reagents were used as received.

\section{Synthesis Of Mn-IONPs}

The Mn-IONPs were prepared following a previously reported procedure with modifications. ${ }^{12}$ The iron-eruciate and manganese-oleate were first synthesized and described in the supporting information (SI) in detail. Next, $2 \mathrm{mmol}$ of iron-eruciate, $1 \mathrm{mmol}$ of manganese-oleate and $6 \mathrm{mmol}$ of oleyl alcohol were dissolved in $10 \mathrm{~mL}$ of dibenzyl ether at room temperature. The mixture was then heated to $100^{\circ}$ $\mathrm{C}$ and maintained for 30 min under a constant argon flow and magnetic stirring. Subsequently, the mixture was heated to $265{ }^{\circ} \mathrm{C}$ at a rate of $5^{\circ} \mathrm{C} / \mathrm{min}$ and was maintained for $1 \mathrm{~h}$. After cooling to room temperature, the synthesized Mn-IONPs were washed with ethanol and centrifuged and were finally dispersed in $20 \mathrm{~mL}$ of hexane for storage.

\section{Surface Modification}

By coating with the DSPE-PEG-2000, the Mn-IONPs were characterized with hydrophilia. Following the previous method, ${ }^{32} 50 \mathrm{mg}$ of Mn-IONPs was first dispersed in 50 $\mathrm{mL}$ of THF to form $1 \mathrm{mg} / \mathrm{mL}$ of Mn-IONPs solution. Next, DSPE-PEG-2000 was mixed with $1 \mathrm{~mL}$ of Mn-IONPs solution with different mass ratios $(\mathrm{NPs} / \mathrm{PEG}=1: 1,1: 5,1: 20$, or $1: 40)$ and then was added dropwise into $5 \mathrm{~mL}$ of deionized water under ultrasonication. After shaking overnight, the supernatants, including Mn-IONPs@PEG (1:1, 1:5, 1:20, or $1: 40$ ), were collected via filtration through a $0.22-\mu \mathrm{m}$ syringe filter and was stored at $4{ }^{\circ} \mathrm{C}$. Additionally, the hydrophilic Mn-IONPs-TMAH were obtained through ligand exchange with tetramethylammonium hydroxide aqueous solution



\section{Characterization}

Transmission electron microscopy (TEM; Hitachi HT7700 , Japan) was used to characterize the morphology of the nanoparticles. High-resolution transmission electron microscopy (HRTEM; FEI TECNAI G20, USA) was used to observe the lattice spacing of the nanoparticles. Powder X-ray diffraction (XRD; PANalytical X'Pert Powder, Netherlands) was performed to detect the structure of the nanoparticles. Inductive-coupled plasma optical emission spectrometry (ICP-OES; Thermo Scientific Icap6300 Duo, USA) was carried out to determine the elemental composition of the nanoparticles. The hysteresis loops of the nanoparticles were recorded using a superconducting quantum interference device (SQUID; VSMVersalab, USA) magnetometer.

\section{Cytotoxicity}

The human liver carcinoma cell line HepG2, purchased from the Chinese Academy of Sciences Cell Bank, was used for cytotoxicity analysis in this study. The cells were routinely cultured in DMEM containing 10\% FBS, $2 \mathrm{mM}$ L-glutamine, and $100 \mathrm{U} / \mathrm{mL}$ penicillin/streptomycin solution in a humidified incubator with $5 \% \mathrm{CO}_{2}$ at $37{ }^{\circ} \mathrm{C}$. CCK-8 was used for the analysis of the cytotoxicity of Mn-IONPs@PEG (1:20). Briefly, the cells were seeded in 96-well plates at a density of $1 \times 10^{4}$ cells/well and were cultured overnight. Next, fresh mediums containing MnIONPs@PEG with serial metal (Fe + Mn) concentrations $(0,1,5,10,20$, or $50 \mu \mathrm{g} / \mathrm{mL})$ were added to replace the previous medium, followed by incubation for an additional $24 \mathrm{~h}$. Each concentration of the samples was repeated five times. Next, the medium was replaced with $100 \mu \mathrm{L}$ of fresh medium including $10 \mu \mathrm{L}$ of WST-8 solution. Following $1.5 \mathrm{~h}$ of coculture, a spectral scanning multimode reader (Thermo Scientific Varioskan Flash, USA) was used to determine the optical density at the wavelength of $450 \mathrm{~nm}$.

\section{MR Imaging In Vitro}

In vitro MR imaging was carried out using a clinical $3.0 \mathrm{~T}$ MRI scanner (GE, Signa HDx 3.0T, USA) with a head coil. The Mn-IONPs@PEG and Mn-IONPs-TMAH (shown in $\underline{\mathrm{SI}}$ ) solutions with [Fe+Mn] concentrations from 0 to $0.5 \mathrm{mM}$ were imaged using the following parameters: fast spin echo sequence (FSE) $\mathrm{T}_{1} \mathrm{WI}$ : repetition time $(\mathrm{TR})=240 \mathrm{~ms}$, echo time $(\mathrm{TE})=7.5 \mathrm{~ms}$, field of view $(\mathrm{FOV})=160 \times 160 \mathrm{~mm}^{2}$, matrix $=320 \times 192$, slice thickness $/$ spacing $=2.0 \mathrm{~mm} / 0.1 \mathrm{~mm}$, number of excitations $(\mathrm{NEX})=4 ; \mathrm{T}_{1}$ mapping: $\mathrm{TE}=9 \mathrm{~ms}, \mathrm{TR}=150,300,600$, $900,1200 \mathrm{~ms}, \mathrm{FOV}=160 \times 160 \mathrm{~mm}^{2}$, matrix $=256 \times 256$, slice thickness/spacing $=2.0 \mathrm{~mm} / 0.1 \mathrm{~mm}, \mathrm{NEX}=1$; FSE $\mathrm{T}_{2} \mathrm{WI}: \mathrm{TR}=2000 \mathrm{~ms}, \quad \mathrm{TE}=46.5 \mathrm{~ms}, \mathrm{FOV}=$ $160 \times 128 \mathrm{~mm}^{2}$, matrix $=192 \times 160$, slice thickness $/$ spacing 
$=2.0 \mathrm{~mm} / 0.1 \mathrm{~mm}, \mathrm{NEX}=4 ; \mathrm{T}_{2}$ mapping: $\mathrm{TR}=1500 \mathrm{~ms}$, $\mathrm{TE}=7.8,15.6,23.5,31.3,39.1,46.9,54.8,62.6 \mathrm{~ms}$, $\mathrm{FOV}=160 \times 128 \mathrm{~mm}^{2}$, matrix $=160 \times 128$, slice thickness $/$ spacing $=2.0 \mathrm{~mm} / 0.4 \mathrm{~mm}$, and $\mathrm{NEX}=1$.

\section{MR Imaging In Vivo}

Animal experiments were conducted in accordance with the National Institutes of Health guidelines on the use of animals in research and were approved by the Laboratory Animal Welfare and Ethics Committee of the Army Medical University. Female C57BL/6 mice with a body weight of approximately $20 \mathrm{~g}$ at the age of 8 weeks were purchased from Chongqing TengXin Biotechnology Co. and were housed at the Experimental Animal Center of XinQiao Hospital. The mice were anesthetized with isoflurane and were maintained in a stereotaxic frame (R510IP; RWD Life Science). The $\mathrm{T}_{1} \mathrm{WI}(\mathrm{TE}=9.3 \mathrm{~ms}, \mathrm{TR}=580 \mathrm{~ms}, \mathrm{FOV}=$ $60 \times 60 \mathrm{~mm}^{2}$, matrix $=288 \times 192$, slice thickness $/$ spacing $=2.0 \mathrm{~mm} / 0 \mathrm{~mm}$, and $\mathrm{NEX}=4)$ and $\mathrm{T}_{2} \mathrm{WI}(\mathrm{TE}=69.6 \mathrm{~ms}$, $\mathrm{TR}=2820 \mathrm{~ms}, \mathrm{FOV}=60 \times 60 \mathrm{~mm}^{2}$, matrix $=288 \times 192$, slice thickness $/$ spacing $=2.0 \mathrm{~mm} / 0 \mathrm{~mm}$, and NEX =6.) Images of mice were acquired using a special rat coil (MS40; Suzhou Medcoil Healthcare Co., Ltd) and a 3.0-T clinical GE MRI scanner before and after injection of the Mn-IONPs@PEG (1:20) with $5 \mathrm{mg}[\mathrm{Fe}+\mathrm{Mn}]$ per kilogram body weight.

\section{Results}

\section{Synthesis And Characterization Of Mn- IONPs}

Mn-IONPs were prepared via thermal decomposition of ironeruciate and manganese-oleate complexes in the presence of oleyl alcohol in dibenzyl ether. Iron-eruciate and manganeseoleate complexes were manufactured through a simple method and are shown in Figure S1. The characteristic bands of ironeruciate appeared at 1582,1557 , and $1433 \mathrm{~cm}^{-1}$, while those of manganese-oleate were at 1606, 1549, and $1423 \mathrm{~cm}^{-1}$, which were consistent with the data of a previous report. ${ }^{12}$ The TEM images and corresponding distribution graphs of the obtained nanoparticles are shown in Figure 1A and B, showing that these nanoparticles were of a size $3.0 \pm 0.28 \mathrm{~nm}$ with a narrow distribution and good uniformity. The crystal lattice fringes shown in the HRTEM images (Figure 1C and D) demonstrated the high crystallinity of the Mn-IONPs, and the measured interplanar distances of 2.56 and $2.97 \AA$ were equivalent to the (311) and (220) lattice planes of $\mathrm{Fe}_{3} \mathrm{O}_{4}$, respectively. The XRD patterns are shown in Figure 1E. Compared with the spinel $\mathrm{Fe}_{3} \mathrm{O}_{4}$ powder diffraction data
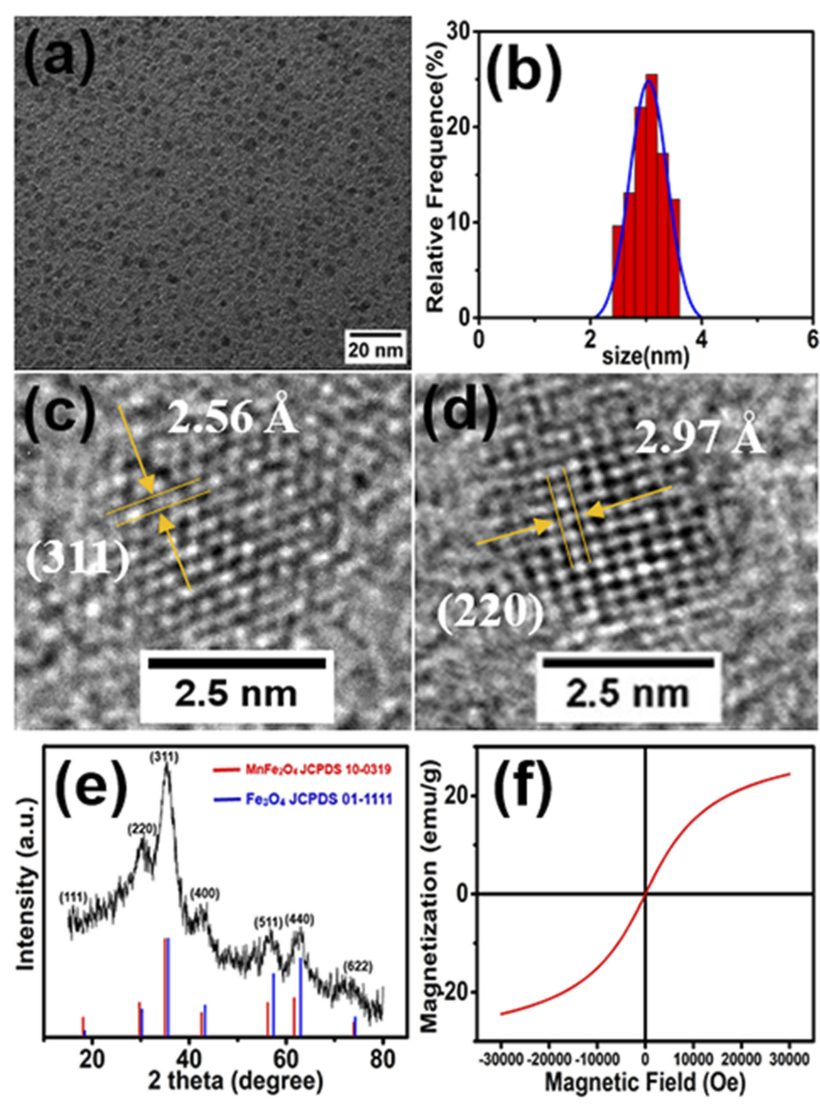

Figure I (A) TEM image, (B) size distribution graph, (C, D) HRTEM images, (E) XRD pattern, and (F) hysteresis loop of synthesized Mn-IONPs.

(JCPDS card no. 01-1111), these diffraction peaks shifted slightly toward that of $\mathrm{MnFe}_{2} \mathrm{O}_{4}$ (JCPDS card no. 10-0319), which can be attributed to the doped manganese. Coupled with the results of ICP-OES showing that the $\mathrm{Mn} / \mathrm{Fe}$ molar ratio was approximately 0.20 , Mn-IONPs were revealed to be successfully prepared. The hysteresis loop recorded by a SQUID magnetometer using a magnetic field up to $3.0 \mathrm{~T}$ at the temperature of $300 \mathrm{~K}$ showed that the saturation magnetization (Ms) of Mn-IONPs was $24.45 \mathrm{emu}^{-1}$ (Figure 1F). Meanwhile, the negligible coercivity and remanence when the external magnetic field was absent indicate their superparamagnetic behavior. Furthermore, after coating with DSPE-PEG-2000 or TMAH, the obtained hydrophilic MnIONPs@PEG or Mn-IONPs-TMAH still maintained size uniformity and showed good stability in water.

\section{Relaxivity Of Mn-IONPs}

To assess $\mathrm{T}_{1}$ and $\mathrm{T}_{2}$ enhancement, the Mn-IONPs@PEG and Mn-IONPs-TMAH solutions were imaged using a 3.0 T clinical MRI scanner with FSE $\mathrm{T}_{1} \mathrm{WI}, \mathrm{FSE}_{2} \mathrm{WI}, \mathrm{T}_{1}$ mapping and $\mathrm{T}_{2}$ mapping sequences. The two types of Mn-IONPs solutions 
all showed concentration-dependent signal enhancement (Figure 2A-D). With the concentration increasing, the signal intensity tended to be brighter in $\mathrm{T}_{1} \mathrm{WI}$ but gradually grew darker in $\mathrm{T}_{2} \mathrm{WI}$. The linear fitting of the concentration and $1 / \mathrm{T}_{1}$ or $1 / T_{2}$ showed that $r_{1}$ values of Mn-IONPs-TMAH, Mn-IONPs@PEG (1:1), Mn-IONPs@PEG (1:5), Mn-IONPs @PEG (1:20), and Mn-IONPs@PEG (1:40)were 3.7, 2.4, 3.5,
7.1, and $7.8 \mathrm{mM}^{-1} \mathrm{~s}^{-1}$, respectively (Figure 2E), and the $r_{2}$ values were $36.9,199.5,149.7,120.9$, and $94.8 \mathrm{mM}^{-1} \mathrm{~s}^{-1}$, respectively (Figure 2F).

\section{In Vitro Cytotoxicity Assay}

Excellent biocompatibility was considered to be an important requirement of Mn-IONPs@PEG for in vivo (a)

$[\mathrm{Fe}+\mathrm{Mn}](\mathrm{mM})$

TMAH

1:1

1:5

$1: 20$

1:40

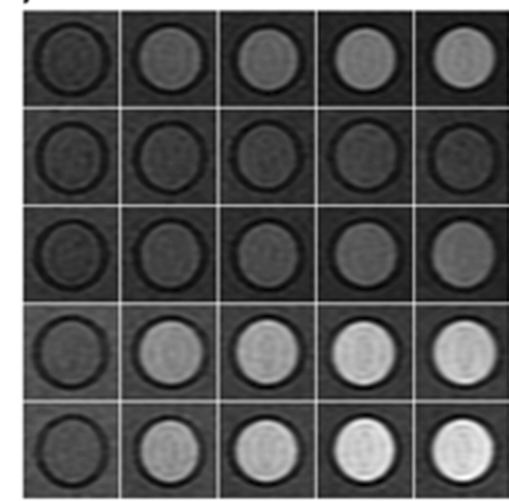

(b)

$\left[\begin{array}{llllll}{[\mathrm{Fe}+\mathrm{Mn}](\mathrm{mM})} & 0 & 0.07 & 0.13 & 0.25 & 0.5\end{array}\right.$

TMAH

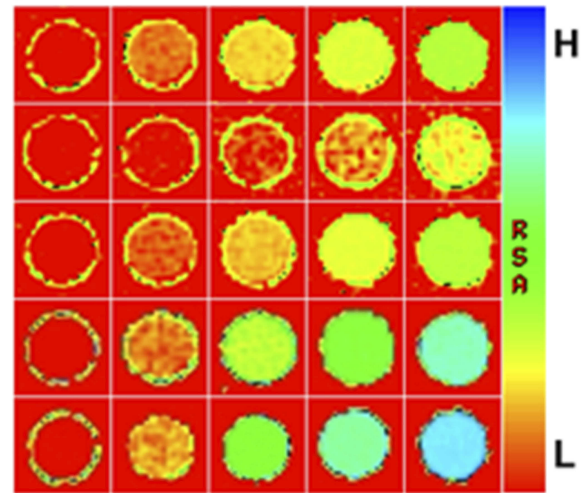

(c) $[\mathrm{Fe}+\mathrm{Mn}](\mathrm{mM})$

0

$\begin{array}{lll}0.07 & 0.13 & 0.25\end{array}$

\section{TMAH}

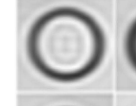

1:1

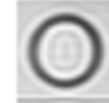

1:5

$1: 20$

$1: 40$

(e)

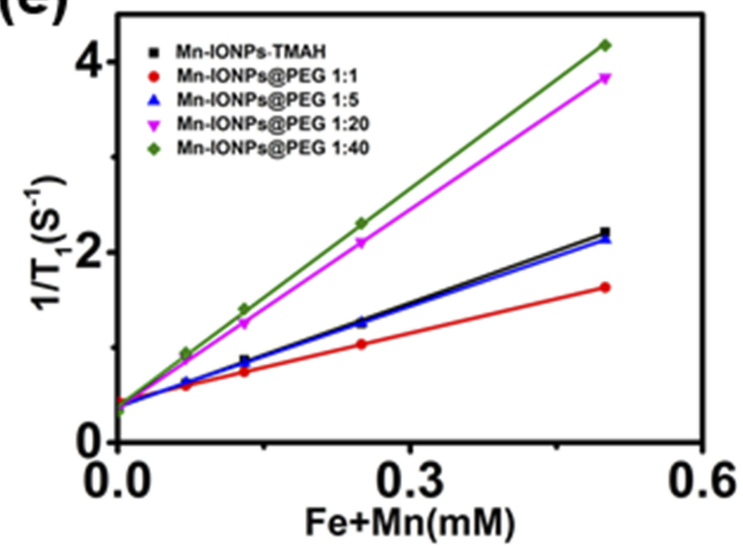

0.5

(d)

\section{1:1}

1:5

$1: 20$

$1: 40$

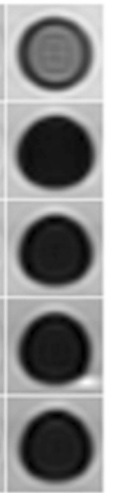

$[\mathrm{Fe}+\mathrm{Mn}](\mathrm{mM})$

TMAH

1:1

1:5

$1: 20$

$1: 40$

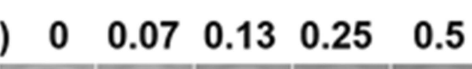

$\begin{array}{llllll}0 & 0.07 & 0.13 & 0.25 & 0.5\end{array}$

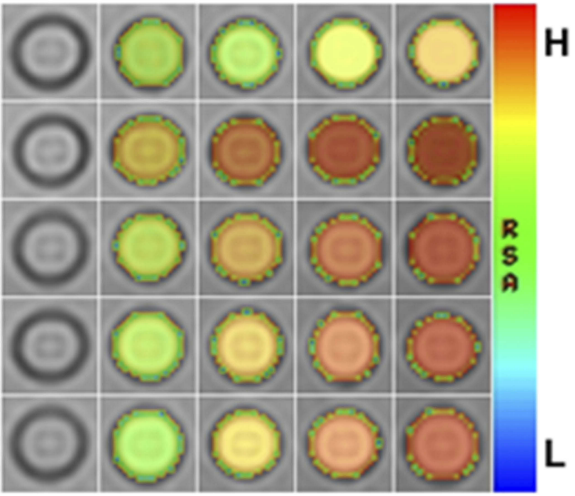

(f)

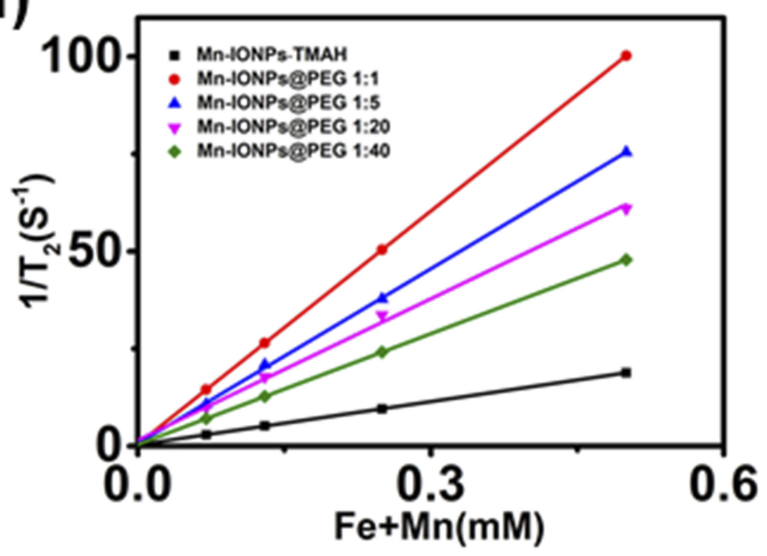

Figure 2 In vitro MR images of the Mn-IONPs-TMAH and Mn-IONPs@PEG (I:I, I:5, I:20, and I:40) at 3.0 T: (A) $T_{1}$-weighted, (B) T, mapping, (C) $T_{2}$-weighted and (D) T2 mapping. (E, F) Linear fitting of $\mathrm{I} / \mathrm{T}_{1}$ and $\mathrm{I} / \mathrm{T}_{2}$ over different $(\mathrm{Fe}+\mathrm{Mn})$ concentrations of the nanocomposites. 
application. The in vitro cell cytotoxicity assay of MnIONPs@PEG (1:20)was performed on HepG2 cells using CCK-8. Figure 3 shows the viability of HepG2 cells cocultured with the Mn-IONPs@PEG of [Fe+Mn] concentration ranging from 1 to $50 \mu \mathrm{g} / \mathrm{mL}$ at $37{ }^{\circ} \mathrm{C}$ for $12 \mathrm{hrs}$ and $24 \mathrm{hrs}$. All the cells survived under the current experimental conditions. All Mn-IONPs@PEG samples showed insignificant toxicity with a cell viability greater than $80 \%$ at a concentration of less than $50 \mu \mathrm{g} / \mathrm{mL}$ for $12 \mathrm{hrs}$ and 24 hrs. These results demonstrated that Mn-IONPs@PEG have a good biocompatibility.

\section{In Vivo Contrast-Enhanced MRI}

Based on the above results, we further explored the potential of the Mn-IONPs as dual-contrast CA in vivo. Due to the high relaxivity, Mn-IONPs@PEG (1:20) were employed for contrast-enhanced MRI of the liver. Figure $4 \mathrm{~A}$ shows the axial $\mathrm{T}_{1} \mathrm{WI}$ and $\mathrm{T}_{2} \mathrm{WI}$ MR images acquired preinjection and postinjection $(0,10,30,60$, and 120 min) of Mn-IONPs@PEG. The significantly brighter $\mathrm{T}_{1} \mathrm{WI}$ MR images and darker $\mathrm{T}_{2} \mathrm{WI}$ MR images suggest good $r_{1}$ and $r_{2}$ values of Mn-IONPs@PEG in vivo. The contrast-enhancement ratios of the MR signal intensity $\Delta \mathrm{SI}\left(\Delta \mathrm{SI}=\left(\mathrm{SI}_{\text {post }}-\mathrm{SI}_{\text {pre }}\right) / \mathrm{SI}_{\text {pre }} \times 100 \%\right)$ are shown in Figure $4 \mathrm{~B}$ and $\mathrm{C}$. The $\Delta$ SI peak of $133 \%$ in $\mathrm{T}_{1} \mathrm{WI}$ appeared at $10 \mathrm{~min}$ after injection followed by a gradual decrease, unexpectedly becoming negative at $120 \mathrm{~min}$. By contrast, the $T_{2}$ WI images showed obvious enhancement at $10 \mathrm{~min}$ after injection but increased to a plateau at $30 \mathrm{~min}$ and maintained for a long period.

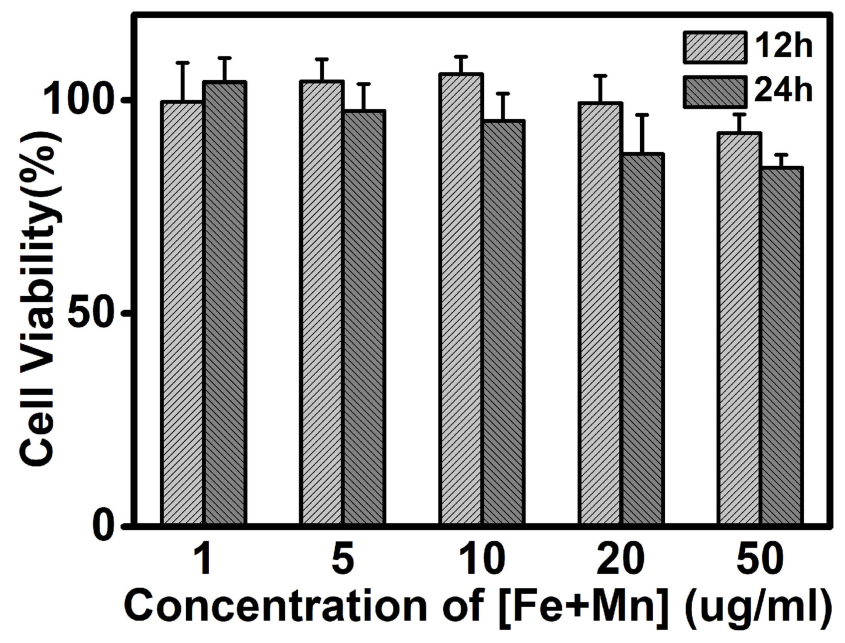

Figure 3 Viability of HepG2 cells after incubation with Mn-IONPs@PEG (I:20) at different concentrations $(\mathrm{I}-50 \mu \mathrm{g} / \mathrm{mL}$ of $[\mathrm{Fe}+\mathrm{Mn}])$ at $37^{\circ} \mathrm{C}$ for $12 \mathrm{hrs}$ and $24 \mathrm{hrs}$.

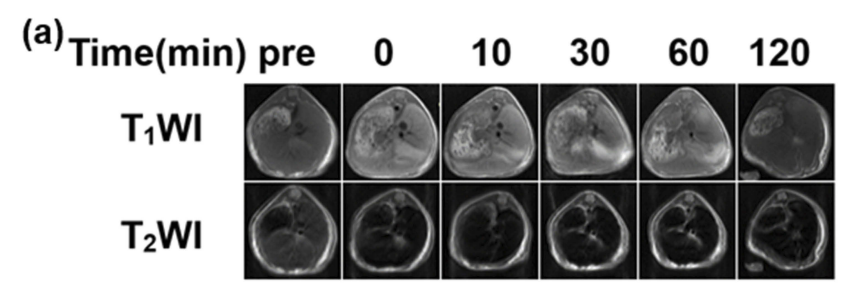

(b)

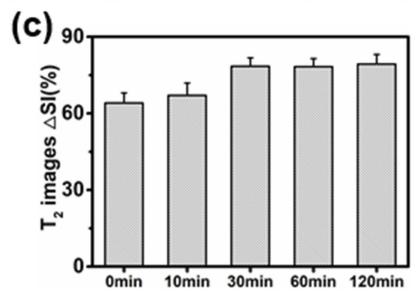

Figure 4 (A) $T_{1}$-weighted and $T_{2}$-weighted $M R$ images of transverse planes of the liver using the FSE sequence acquired at $0,10,30,60$, or $120 \mathrm{~min}$ after intravenous administration of Mn-IONPs@PEG (I:20). (B, C) Enhancement ratio of the MR signal $\Delta \mathrm{SI}$ of the liver after contrast-enhancement by Mn-IONPs@PEG (I:20).

\section{Discussion}

As is well known, $T_{1}$ enhancement of superparamagnetic NPs depends on the interaction of the paramagnetic ions on the surface and nearby proton. Doping paramagnetic ions into the NPs has proven to be an effective route to improve $r_{1}{ }^{29}$ Many kinds of metal ions have been reported to be paramagnetic because of their unpaired electrons. Unlike lanthanide ions, the transition metal ions show both excellent biocompatibility and comparative paramagnetism and should be considered better ions for doping. $\mathrm{Mn}^{2+}$ with five unpaired electrons shows the highest paramagnetism compared with other transition metal ions. ${ }^{2}$ Additionally, based on the quantum mechanical inner-sphere theory, the $T_{1}$ relaxivity for NPs-based $T_{1}$ CA can be given by ${ }^{33}$

$$
\frac{1}{\mathrm{~T}_{1}}=\frac{q P_{M}}{\mathrm{~T}_{1 M}+\tau_{M}}
$$

where $P_{M}$ is the mole fraction of metal ions, $q$ is the number of water molecules bound per metal ion, $\tau_{M}$ is the residence lifetime of the bound water, and $\mathrm{T}_{1 M}$, which is related to $\tau_{M}$ and the rotational correlation time $\left(\tau_{R}\right)$, is the relaxation time of the bound water protons. It was revealed that the $\tau_{M}$ of $\mathrm{Mn}$ $\left(\mathrm{H}_{2} \mathrm{O}\right)_{6}{ }^{2+}$ is $4.3 \times 10^{4} \mathrm{~ns}$ and is 2 orders of magnitude smaller than that of $\mathrm{Fe}\left(\mathrm{H}_{2} \mathrm{O}\right)_{6}{ }^{3+}\left(5.3 \times 10^{6} \mathrm{~ns}\right)$ and other transition metal ions. ${ }^{33}$ Thus, $\mathrm{Mn}^{2+}$ doping will effectively accelerate spin-lattice relaxation and increase $r_{1}$. Moreover, embedding paramagnetic ions into the superparamagnetic NPs significantly boosts both $r_{1}$ and $r_{2}$ of superparamagnetic hybrid NPs by synergetic interaction between the $\mathrm{T}_{1}$ and $\mathrm{T}_{2}$ species. ${ }^{16}$

In addition to $\mathrm{Mn}^{2+}$ doping, decreasing the size of NPs into the ultrasmall region has also been proven to be an effective approach to boost $r_{1}$. The smaller the size of 
superparamagnetic NPs is, the larger the surface-tovolume ratio is, and the more superficial paramagnetic ions are, and the better $r_{1}$ the NPs possess. ${ }^{12}$ Thus, sufficiently small $\mathrm{Mn}^{2+}$ doping nanoparticles must be synthesized to guarantee good $r_{1}$. Theoretically, the key to successfully obtain ultrasmall $\mathrm{Mn}^{2+}$ doping nanoparticles is to decompose both the precursors at a similar temperature to form a multiple metal-containing monomer before nucleation to obtain smaller nanoparticles instead of a single-metal-containing monomer, resulting in the formation of a nondopant-containing nucleus initially and then another metal serving as the host to grow nanoparticles. ${ }^{12}$ Thus, the Mn-IONPs in this study were prepared by DSTD of the iron-eruciate and manganese-oleate complexes, which had a similar decomposition temperature in the presence of oleyl alcohol in dibenzyl ether instead of iron (III) acetylacetonate and manganese (II) acetylacetonate. From the results of TEM, HRTEM, XRD and ICP, Mn-IONPs with an ultrasmall size of $3.0 \pm 0.28 \mathrm{~nm}$ were successfully synthesized and the $\mathrm{Mn}^{2+}$ was fully proven to be doped successfully.

It is very important to have both good $r_{1}$ and $r_{2}$ to be good $\mathrm{T}_{1} / \mathrm{T}_{2}$ dual-contrast CA. However, we decreased the size of the NPs to the ultrasmall range to reach an optimal $r_{1}$ of the NPs in this study, resulting in a relative thicker spin-canted layer and a much lower saturation magnetization $(M s)$ and, finally, a much lower $r_{2}$ as expected. Unlike $r_{1}$, the relaxation of $\mathrm{T}_{2} \mathrm{CA}$ is dominated by the outersphere mechanism and can be given by ${ }^{34}$

$$
\frac{1}{\mathrm{~T}_{2}}=\frac{256 \pi^{2} \gamma_{I} M_{n}}{1215 \rho}\left(\frac{1}{1+L / a}\right)^{3} M s^{2} \tau_{D}
$$

where $\gamma_{I}, M_{n}, \rho, a$ and $L$ are the proton gyromagnetic ratio, molar mass, density, radius of NPs and impermeable thickness of the surface coating layer, respectively. $\tau_{D}$ is the translational diffusion time, which is determined by $r$ and $D$ according to the equation $\tau_{D}=r^{2} / D$, where $r$ and $D$ are the effective radius and diffusion coefficient, respectively. It is obvious that $r_{2}$ is proportional to $a^{3}$, while $M s^{2}$ and $\tau_{D}$ are inversely proportional to $L^{3}$. To increase $M s$, we doped $\mathrm{Mn}^{2+}$ into ultrasmall supermagnetic magnetite. Because the doped $\mathrm{Mn}^{2+}$ can occupy both the tetrahedral $\left(\mathrm{T}_{\mathrm{d}}\right)$ and octahedral holes $\left(\mathrm{O}_{\mathrm{h}}\right)$ in the crystal lattices, Mn-IONPs form a special mixed spinel structure, finally resulting in larger $M s$ and $r_{2}$; this finding has been confirmed in this study by showing that the $r_{2}$ of Mn-IONPs-TMAH (36.9 $\left.\mathrm{mM}^{-1} \mathrm{~s}^{-1}\right)$ is larger than IONPs with the same size $\left(r_{2}=29.2 \mathrm{mM}^{-1} \mathrm{~s}^{-1}\right) .{ }^{15}$ Using another approach by coating Mn-IONPs with DSPE-PEG, we further enhance its $r_{2}$.

According to Equation (2), $r_{2}$ can be improved by decreasing $D$. The PEG molecules on the surface of the Mn-IONPs can immobilize surrounding water molecules in a large region by forming hydrogen bonds, resulting in a small $D$ and a large $\tau_{D} \cdot{ }^{30,31}$ Thus, it is reasonable that all the DSPE-PEG-coated Mn-IONPs showed a much higher $r_{2}$ than THAM-coated Mn-IONPs. However, a decreasing trend for $r_{2}$ was noted with the increase in the molar ratio of Mn-IONPs: DSPE-PEG. This phenomenon can be easily explained because the hydrogen-bond interactions between PEG and water protons have a critical molar ratio. Too many coating molecules may exclude water protons from the induced magnetic field from the Mn-IONPs, forming an impermeable coating layer on the surface of the Mn-IONPs and leading to a lower $r_{2}$. Interestingly, we also found that $r_{1}$ can be changed by varying the coating molecules and the molar ratio of these molecules. MnIONPs-TMAH showed a higher $r_{1}$ than Mn-IONPs-PEG (1:1) and Mn-IONPs-PEG (1:5) but possessed a lower $r_{1}$ than Mn-IONPs-PEG (1:20) and Mn-IONPs-PEG (1:40). Unlike $r_{2}, r_{1}$ increased significantly with the increase in the molar ratio of Mn-IONPs: DSPE-PEG. This phenomenon can be attributed to $\tau_{R}$ and $q$ based on Equation (1). As is well known, $T_{1}$ relaxation relies on a chemical exchange between the paramagnetic center and water molecules in the first coordination sphere. The more water molecules located in the first coordination sphere, the better $r_{1}$ is. Although NPs become hydrophilic by coating with DSPE-PEG, there is still a DSPE-oleic acid layer that is impermeable on the surface of the NPs, resulting in much fewer water molecules in the first coordination sphere than Mn-IONPs-TMAH, in which the impermeable oleic acid layer has been exchanged. Thus, the $r_{1}$ of Mn-IONPs-TMAH was higher than that of MnIONPs-PEG (1:1) and Mn-IONPs-PEG (1:5). However, why do Mn-IONPs-PEG (1:20) and Mn-IONPs-PEG (1:40) show a higher $r_{1}$ than Mn-IONPs-TMAH? The cause may be that $r_{1}$ can also be remarkably influenced by $\tau_{R}$, which is proportional to the molecular weight. With the increase in the molar ratio of Mn-IONPs: DSPE-PEG, the molecular weight of NPs increases notably, leading to a higher $r_{1}$. Over the critical ratio, the shielding effect of the impermeable DSPE-oleic acid layer will be counteracted and $\tau_{R}$ will be dominant, making the $r_{1}$ of MnIONPs-PEG (1:20) and Mn-IONPs-PEG (1:40) higher than that of Mn-IONPs-TMAH. 
Excellent biocompatibility is considered a key point for inorganic NPs application in vivo. ${ }^{11}$ To improve the biocompatibility, we selected DSPE-PEG, a well-established nonimmunogenic and noncytotoxic material, for Mn-IONPs coating. By CCK-8 analysis, the acquired MnIONPs@PEG (1:20) showed negligible cytotoxicity and excellent biocompatibility, which were consistent with the results of previous studies. ${ }^{30,31,35}$ Thus, we further explored the potential of Mn-IONPs@PEG (1:20) for $T_{1} / T_{2}$ dualcontrast MR imaging in vivo. Obviously, clear signal intensity changes were noted in both $\mathrm{T}_{1} \mathrm{WI}$ and $\mathrm{T}_{2} \mathrm{WI}$ over time. The signal intensity changes in the liver may be the result of the biological distribution and metabolism of $\mathrm{Mn}$ IONPs@PEG in vivo. After injection, Mn-IONPs@PEG gradually accumulated in the liver within 10 mins, leading to increased $\Delta$ SI of both $T_{1}$ and $T_{2}$. However, the $\Delta$ SI of $\mathrm{T}_{1} \mathrm{WI}$ decreased over time because of passive aggregation driven by the phagocytosis of Kupffer cells, resulting in stable and individual Mn-IONPs@PEG gradually aggregating into clusters and causing the larger $\mathrm{T}_{2}$ contrast enhancement compared with the smaller $\mathrm{T}_{1}$ contrast enhancement. Particularly, the $\Delta \mathrm{SI}$ even turned out to be negative at 120 min because the dominated $T_{2}$ contrast enhancement significantly affected the $T_{1}$ contrast. By contrast, the $\Delta$ SI of $\mathrm{T}_{2}$ WI kept increasing until it reached a plateau and there was no downward trend within the observation period because of no degradation. The results obtained from the in vivo MRI verified that Mn-IONPs@PEG are highly sensitive $T_{1}$ and $T_{2}$ dual $C A$ for imaging of the liver.

\section{Conclusion}

In summary, we successfully synthesized 3-nm-sized MnIONPs via facile thermal decomposition of iron-eruciate and manganese-oleate complexes in the presence of oleyl alcohol in dibenzyl ether. After encapsulation in DSPEPEG-2000, the Mn-IONPs@PEG displayed good colloidal stability and excellent biocompatibility. More importantly, due to $\mathrm{Mn}^{2+}$ doping and DSPE-PEG-2000 coating, the synthesized NPs showed unexpected high $r_{1}$ and $r_{2}$. Confirmed by in vitro and in vivo MR imaging, the MnIONPs@PEG are ideal candidates for use as $\mathrm{T}_{1} / \mathrm{T}_{2}$ dualcontrast CA.

\section{Acknowledgment}

This research was supported by National Science Foundation of China (No. 81471635 and 81501521), Natural Science Foundation of Chongqing (No. cstc2018jcyjAX0321 and cstc2017jcyjBX0038).

\section{Disclosure}

The authors report no conflicts of interest in this work.

\section{References}

1. Smith BR, Gambhir SS. Nanomaterials for in vivo imaging. Chem Rev. 2017;117(3):901-986. doi:10.1021/acs.chemrev.6b00073

2. Ni D, Bu W, Ehlerding EB, et al. Engineering of inorganic nanoparticles as magnetic resonance imaging contrast agents. Chem Soc Rev. 2017;46(23):7438-7468. doi:10.1039/c7cs00316a

3. Weissleder R. Molecular imaging in cancer. Science. 2006; 312:1168-1171.

4. Weissleder R. Molecular imaging: exploring the next frontier. Radiology. 1999;212(3):609-614.

5. Herschman HR. Molecular imaging: looking at problems, seeing solutions. Science. 2003;302(5645):605-608.

6. Louie AY, Hüber MM, Ahrens ET, et al. In vivo visualization of gene expression using magnetic resonance imaging. Nat Biotechnol. 2000;18:321.

7. Liu J, Wang L, Cao J, et al. Functional investigations on embryonic stem cells labeled with clinically translatable iron oxide nanoparticles. Nanoscale. 2014;6(15):9025-9033.

8. Willmann JK, van Bruggen N, Dinkelborg LM, Gambhir SS. Molecular imaging in drug development. Nat Rev Drug Discov. 2008;7(7):591-607.

9. Yang Z, Ren J, Ye Z, et al. Bio-inspired synthesis of PEGylated polypyrrole@polydopamine nanocomposite as theranostic agent for $\mathrm{t}_{1}$-weighted MR imaging guided photothermal therapy. $J$ Mater Chem B. 2017;5:1108-1116.

10. Zhang F, Ni Q, Jacobson O, et al. Polymeric nanoparticles with a glutathione-sensitive heterodimeric multifunctional prodrug for in vivo drug monitoring and synergistic cancer therapy. Angew Chem Int Ed Engl. 2018;57(24):7066-7070. doi:10.1002/anie.201801984

11. Na HB, Song IC, Hyeon T. Inorganic nanoparticles for MRI contrast agents. Adv Mater. 2009;21(21):2133-2148. doi:10.1002/adma.v21:21

12. Zhang H, Li L, Liu XL, et al. Ultrasmall ferrite nanoparticles synthesized via dynamic simultaneous thermal decomposition for highperformance and multifunctional $\mathrm{T}_{1}$ magnetic resonance imaging contrast agent. ACS Nano. 2017;11(4):3614-3631. doi:10.1021/ acsnano.6b07684

13. Jang JT, Nah H, Lee JH, et al. Critical enhancements of MRI contrast and hyperthermic effects by dopant-controlled magnetic nanoparticles. Angew Chem Int Ed Engl. 2009;48(7):1234-1238. doi:10.1002/ anie. 200805149

14. Shin T-H, Choi J-S, Yun $\mathrm{S}$, et al. $\mathrm{T}_{1}$ and $\mathrm{T}_{2}$ dual-mode MRI contrast agent for enhancing accuracy by engineered nanomaterials. ACS Nano. 2014;8:3393-3401. doi:10.1021/nn405977t

15. Kim BH, Lee N, Kim H, et al. Large-scale synthesis of uniform and extremely small-sized iron oxide nanoparticles for high-resolution $T_{1}$ magnetic resonance imaging contrast agents. $J$ Am Chem Soc. 2011;133(32):12624-12631. doi:10.1021/ja203340u

16. Zhou Z, Huang D, Bao J, et al. A synergistically enhanced $T_{1}-T_{2}$ dual-modal contrast agent. Adv Mater. 2012;24(46):6223-6228. doi:10.1002/adma.201203169

17. Li J, You J, Wu C, et al. $\mathrm{T}_{1}-\mathrm{T}_{2}$ molecular magnetic resonance imaging of renal carcinoma cells based on nano-contrast agents. Int J Nanomedicine. 2018;13:4607-4625. doi:10.2147/IJN.S168660

18. Im GH, Kim SM, Lee DG, et al. $\mathrm{Fe}_{3} \mathrm{O}_{4} / \mathrm{MnO}$ hybrid nanocrystals as a dual contrast agent for both $\mathrm{T}_{1^{-}}$and $\mathrm{T}_{2}$-weighted liver MRI. Biomaterials. 2013;34(8):2069-2076. doi:10.1016/j.biomaterials.2012.11.054

19. Tegafaw T, Xu W, Ahmad MW, et al. Dual-mode $T_{1}$ and $T_{2}$ magnetic resonance imaging contrast agent based on ultrasmall mixed gadolinium-dysprosium oxide nanoparticles: synthesis, characterization, and in vivo application. Nanotechnology. 2015;26(36):365102. doi:10.1088/0957-4484/26/36/365102 
20. Yang M, Gao L, Liu K, et al. Characterization of $\mathrm{Fe}_{3} \mathrm{O}_{4} / \mathrm{SiO}_{2} / \mathrm{Gd}_{2} \mathrm{O}\left(\mathrm{CO}_{3}\right)_{2}$ core/shell/shell nanoparticles as $\mathrm{T}_{1}$ and $\mathrm{T}_{2}$ dual mode MRI contrast agent. Talanta. 2015;131:661-665. doi:10.1016/j.talanta.2014.08.042

21. Sun X, Du R, Zhang L, et al. A pH-responsive yolk-like nanoplatform for tumor targeted dual-mode magnetic resonance imaging and chemotherapy. ACS Nano. 2017;11(7):7049-7059. doi:10.1021/acsnano.7b02675

22. Cabrera-Garcia A, Checa-Chavarria E, Pacheco-Torres J, et al. Engineered contrast agents in a single structure for $\mathrm{T}_{1}-\mathrm{T}_{2}$ dual magnetic resonance imaging. Nanoscale. 2018;10(14):6349-6360. doi:10.1039/C7NR07948F

23. Gong $M$, Yang $H$, Zhang $S$, et al. Targeting $T_{1}$ and $T_{2}$ dual modality enhanced magnetic resonance imaging of tumor vascular endothelial cells based on peptides-conjugated manganese ferrite nanomicelles. Int J Nanomedicine. 2016;11:4051-4063.

24. Lee N, Hyeon T. Designed synthesis of uniformly sized iron oxide nanoparticles for efficient magnetic resonance imaging contrast agents. Chem Soc Rev. 2012;41(7):2575-2589.

25. Wang G, Zhang X, Skallberg A, et al. One-step synthesis of waterdispersible ultra-small $\mathrm{Fe}_{3} \mathrm{O}_{4}$ nanoparticles as contrast agents for $\mathrm{T}_{1}$ and $\mathrm{T}_{2}$ magnetic resonance imaging. Nanoscale. 2014;6(5):2953-2963.

26. Pellico J, Ruiz-Cabello J, Fernandez-Barahona I, et al. One-step fast synthesis of nanoparticles for MRI: coating chemistry as the key variable determining positive or negative contrast. Langmuir. 2017;33(39):10239-10247.

27. Li Z, Wang SX, Sun Q, et al. Ultrasmall manganese ferrite nanoparticles as positive contrast agent for magnetic resonance imaging. $A d v$ Healthc Mater. 2013;2(7):958-964.
28. Zhang M, Cao Y, Wang L, et al. Manganese doped iron oxide theranostic nanoparticles for combined $\mathrm{T}_{1}$ magnetic resonance imaging and photothermal therapy. ACS Appl Mater Interfaces. 2015;7 (8):4650-4658.

29. Lee JH, Huh YM, Jun YW, et al. Artificially engineered magnetic nanoparticles for ultra-sensitive molecular imaging. Nat Med. 2007;13(1):95-99.

30. Tong S, Hou S, Zheng Z, et al. Coating optimization of superparamagnetic iron oxide nanoparticles for high $\mathrm{T}_{2}$ relaxivity. Nano Lett. 2010;10(11):4607-4613.

31. LaConte LE, Nitin N, Zurkiya O, et al. Coating thickness of magnetic iron oxide nanoparticles affects $\mathrm{R}_{2}$ relaxivity. J Magn Reson Imaging. 2007;26(6):1634-1641.

32. Lu J, Ma S, Sun J, et al. Manganese ferrite nanoparticle micellar nanocomposites as MRI contrast agent for liver imaging. Biomaterials. 2009;30(15):2919-2928.

33. RB LAUFFER. Paramagnetic metal complexes as water proton relaxation agents for NMR imaging theory and design. Chem Rev. 1987;87:901-927.

34. Zeng J, Jing L, Hou Y, et al. Anchoring group effects of surface ligands on magnetic properties of $\mathrm{Fe}_{3} \mathrm{O}_{4}$ nanoparticles: towards high performance MRI contrast agents. Adv Mater. 2014;26(17):26942698. 2016

35. Johnson NJ, He S, Nguyen Huu VA, Almutairi A. Compact micellization: a strategy for ultrahigh $\mathrm{t}_{1}$ magnetic resonance contrast with gadolinium-based nanocrystals. ACS Nano. 2016;10(9):8299-8307.
International Journal of Nanomedicine

\section{Publish your work in this journal}

The International Journal of Nanomedicine is an international, peerreviewed journal focusing on the application of nanotechnology in diagnostics, therapeutics, and drug delivery systems throughout the biomedical field. This journal is indexed on PubMed Central, MedLine, CAS, SciSearch ${ }^{\circledR}$, Current Contents ${ }^{\circledR} /$ Clinical Medicine,

\section{Dovepress}

Journal Citation Reports/Science Edition, EMBase, Scopus and the Elsevier Bibliographic databases. The manuscript management system is completely online and includes a very quick and fair peer-review system, which is all easy to use. Visit http://www.dovepress.com/ testimonials.php to read real quotes from published authors. 